\title{
TEACHING TARGET VALUE DESIGN FOR DIGITAL FABRICATION IN AN ONLINE GAME: OVERVIEW AND CASE STUDY
}

\author{
Ming Shan $\mathrm{Ng}^{1}$ and Daniel Mark Hall ${ }^{2}$
}

\begin{abstract}
Digital fabrication (DFAB) for construction automation is emerging in the industry. However, DFAB requires better integration of fabrication-related information and organisation into the design process. Discrete processes in traditional delivery models such as Design-Bid-Build can hinder DFAB implementation when stakeholders find it hard to manage project costs. Target Value Design (TVD) has been proposed as possible approach to manage the DFAB design process, but management of DFAB using TVD is still new in the industry. Meanwhile, existing educational games have been successful at teaching players the basic principles of TVD principles. However, these games do not explicitly consider how players should select from advanced fabrication processes. They also have not yet been adopted for online play. This work presents an overview of an online TVD for DFAB game that can 1) help players understand basic TVD principles and 2) explicitly considers fabrication processes and resulting production times as an additional project value. The paper presents the results of a validation case played by 36 construction professionals, researchers and students in December 2020. Overall, this work contributes to the body of knowledge in learning and teaching TVD, online lean games, and technology adoption.
\end{abstract}

\section{KEYWORDS}

Target Value Design (TVD), digital fabrication, target cost, collaboration, concurrent, integrated project delivery, design management.

\section{INTRODUCTION}

Digital fabrication (DFAB) is emerging as a systemic innovation to foster automation and boost productivity in the construction industry (Agarwal et al. 2016). However, DFAB has not yet been widely adopted in projects. A key barrier that hinders DFAB adoption is the sceptical attitude from project stakeholders to manage DFAB in construction projects (Carra et al. 2018). Recent research finds that DFAB transforms design and construction processes and therefore requires better integration of fabrication-related information and organisation (Bock and Linner 2015; Hall et al. 2019; Ng et al. 2020). With traditional project delivery models such as Design-Bid-Build (DBB), information, organisation and

Scientific Researcher, Chair of Innovative and Industrial Construction, Department of Civil, Environmental and Geomatic Engineering, ETH Zurich, Stefano-Franscini-Platz 5, 8093 Zurich, Switzerland, ng@ibi.baug.ethz.ch, orcid.org/0000-0003-2610-3561

2 Assistant Professor, Chair of Innovative and Industrial Construction, Department of Civil, Environmental and Geomatic Engineering, ETH Zurich, Stefano-Franscini-Platz 5, 8093 Zurich, Switzerland, dhall@ethz.ch, orcid.org/0000-0002-0957-484X 
process are discrete in design and construction. Stakeholders find it hard to manage project values such as costs and schedule without the knowledge of the construction process, in particular for management for novel technology implementation (Ballard 2011; Carra et al. 2018).

DFAB adoption could benefit from innovative design management approach such as the lean-based Target Value Design (TVD). TVD is lean-based approach that involves design based on detailed cost estimates (Macomber et al. 2007). TVD enables concurrent engineering, design-to-target-values and maximise values to project stakeholders (Ballard and Morris 2010; Miron et al. 2015; Tommelein and Ballard 2016; Ng and Hall 2019). However, TVD requires a radically different design management approach than found in traditional DBB projects. There is a need to educate project stakeholders about the key principles and mechanisms of TVD. To do this, the lean construction community often uses "the Marshmallow game" to teach TVD (Rybkowski et al. 2016). While the Marshmallow game has been very successful and should be considered a foundational building block for teaching TVD, two limitations relevant to this paper should be noted. First, the Marshmallow game does not translate well to an online environment, which was shown to be a need in the time of the COVID-19 pandemic. Second, the Marshmallow game does not explicitly consider the selection among advanced fabrication processes which may have a significant impact on project values such as schedule.

As an alternative, this paper presents the "Target Value Design for Digital Fabrication (TVDfDFAB) online game". This game aims to teach and leverage the use of DFAB in TVD in design. First, the paper presents an overview of the TVDfDFAB game. Next, the results of the validation in a case study undertaken in a master's degree class played by 36 industry experts, researchers and students in December 2020 at ETH Zurich in Switzerland. This is followed by the Discussion section with limitations of the game and this work, as well as the proposed future research. The work-in-progress version of the game has been published online for dissemination since November 2020 and openly accessible with the link in the footnote. ${ }^{3}$

\section{POINT OF DEPARTURE}

\section{DESIGN FOR Digital FABRICATION (DFAB) IN CONSTRUCTION}

DFAB refers to data-driven production which aims to improve productivity and efficiency through automation in fabrication processes (Agarwal et al. 2016). It requires fabrication information to be included in early stages of the design process. However, stakeholders often find it hard to incorporate DFAB information and organisation in discrete design and construction processes in, for example, DBB projects ( $\mathrm{Ng}$ et al. 2020). This can lead to skeptical attitudes about DFAB innovations which can hinder adoption on construction projects (Carra et al. 2018). To address this, researchers have investigated novel design approaches for DFAB such as Design for Automation (DfA) (Bridgewater 1993) and Robot-Oriented Design (Bock and Linner 2015). Ng and Hall (2019) investigate the intersection of lean management with DfMA and DFAB to identify shared practices of concurrent engineering and design-to-target-values. These two keys practices seem critical to foster organisation, information and process integration and maximise values for project stakeholders on DFAB projects (Rybkowski 2009; Ng et al. 2020).

\footnotetext{
${ }^{3}$ The work-in-progress (WIP) version of the game can be accessed here: https://www.researchcollection.ethz.ch/handle/20.500.11850/467162
} 


\section{TARGeT VALUE DeSign (TVD)}

TVD is an adaption of the original Toyota's target costing concept to construction industry (Zimina et al 2012). TVD was introduced to the construction industry by Macomber et al. (2007) and Ballard (2011). In many existing projects, TVD helps to maximise project values during the design process and could result in $15 \%$ to $20 \%$ below market price without compromises in quality and duration of the products and the processes (Rybkowski 2009; Zimina et al. 2012). To assist stakeholders to comprehend and adopt TVD principles in practice, the lean construction community has developed the "Marshmallow Game" presented by Rybkowski et al. (2016). In its 1-hour-20-minute version, players can experience outcome differences between a linear, silo-ed DBB design process in the first round, where no costing goals are specified, and an integrated, co-located TVD process in the second round. The materials to build the towers, time to complete the exercise, the Requests for Information (RFIs) and Change Orders during the design process are calculated. The Marshmallow game demonstrates value management in TVD in comparison with that in DBB design process (Rybkowski et al. 2016).

However, the marshmallow game simplifies the decision-making process to be exclusive of fabrication. Player are free to modify their procured materials in any way they wish with not cost implications. For example, there is no cost implication if players wish to cut up a straw into multiple smaller pieces or keep it as one single piece. While this is an intentional simplification made by the marshmallow game to avoid unneccesary complexity, it also does not reflect the reality of the considerations needed for adoption of DFAB on a construction project. Furthermore, to the authors' knowledge, no TVD games can yet be played online.

\section{GAME DESCRIPTION}

The authors of this work developed this TVDFDFAB game, which aims to assist industry practitioners and students in the construction industry to comprehend TVD principles to manage project values during the design process using DFAB technology in the fabrication. Since DFAB is still in its early stage of implementation in the industry, not many industry practitioners and students have experience in the design process for DFAB. To ensure knowledge of DFAB is not a prerequisite to play the game, the authors adopted the design process in a commercial kitchen scenario. The intended connection between the kitchen scenario and the construction industry is described later in the paper.

To cope with the remote work and online teaching, the TVDfDFAB game can be played online via video conferencing platforms (e.g., Zoom) and with open-access cloudbased documents (in this case, Google Slides and Google Sheets). The game requires a presenter or moderator to control the rundown and to present the presenter's deck throughout the game. The game is composed of two successive rounds similar to the Marshmallow game. Round 1 is intended to reflect the traditional DBB design approach; Round 2 is intended to teach the benefits of the TVD approach. In each round, the players are given a set of the player's deck on Google Slides and the player's spreadsheet on Google Sheets.

\section{ROLES AND DELIVERABLES}

The game requires players to form groups of four. Each player in the group will play the role of an Artistic Chef, a Recipe Chef, an Executive Chef or a Restaurant Owner (Figure 1) throughout the game. In both rounds, all teams have the same goal to design a plate of 
salad for the team's newly opened 4-star restaurant. The scope of work and deliverables of each role are the same in both rounds. The ingredients available to compose the salad are carrot, cucumber, tomato and egg. The slices have been prepared for the Artistic Chefs to select, drag and drop during the design processes. Also, the tools are available in mainly three levels of automation- manual, semi-automated and fully-automated - as listed in three columns for the Executive Chefs to select on the Google Sheet as presented in Figure 2. In both rounds, the customer requests a "best design" salad with (i) at least 500g, the heavier the better; (ii) equally balanced in weights between the given ingredients; and (iii) inspired by the Vincent van Gogh's The Starry Night painting (Figure 3). In Round 1, the customer requests for a good price, while the restaurant profit accounts for $5 \%$ of the total cost. In Round 2, the customer offers a target price, while the restaurant profit is calculated by the set target price minus the total cost. The total cost in both rounds is calculated based on the total process cost with the selected tools, the total process time, penalties for weight imbalance of the ingredients and for underweight and bonus for the extra weight above $500 \mathrm{~g}$.

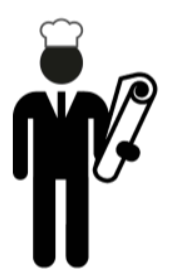

ARTISTIC CHEF

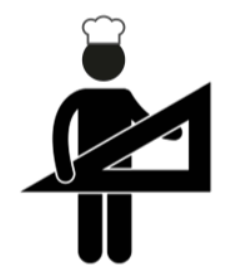

RECIPE CHEF

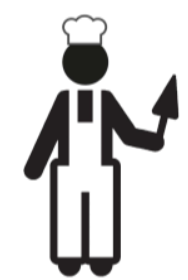

EXECUTIVE CHEF

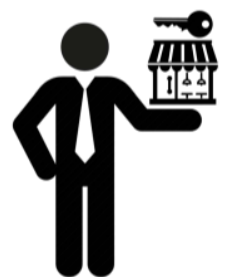

RESTAURANT OWNER

Figure 1: The roles in a team of four in the TVDfDFAB game

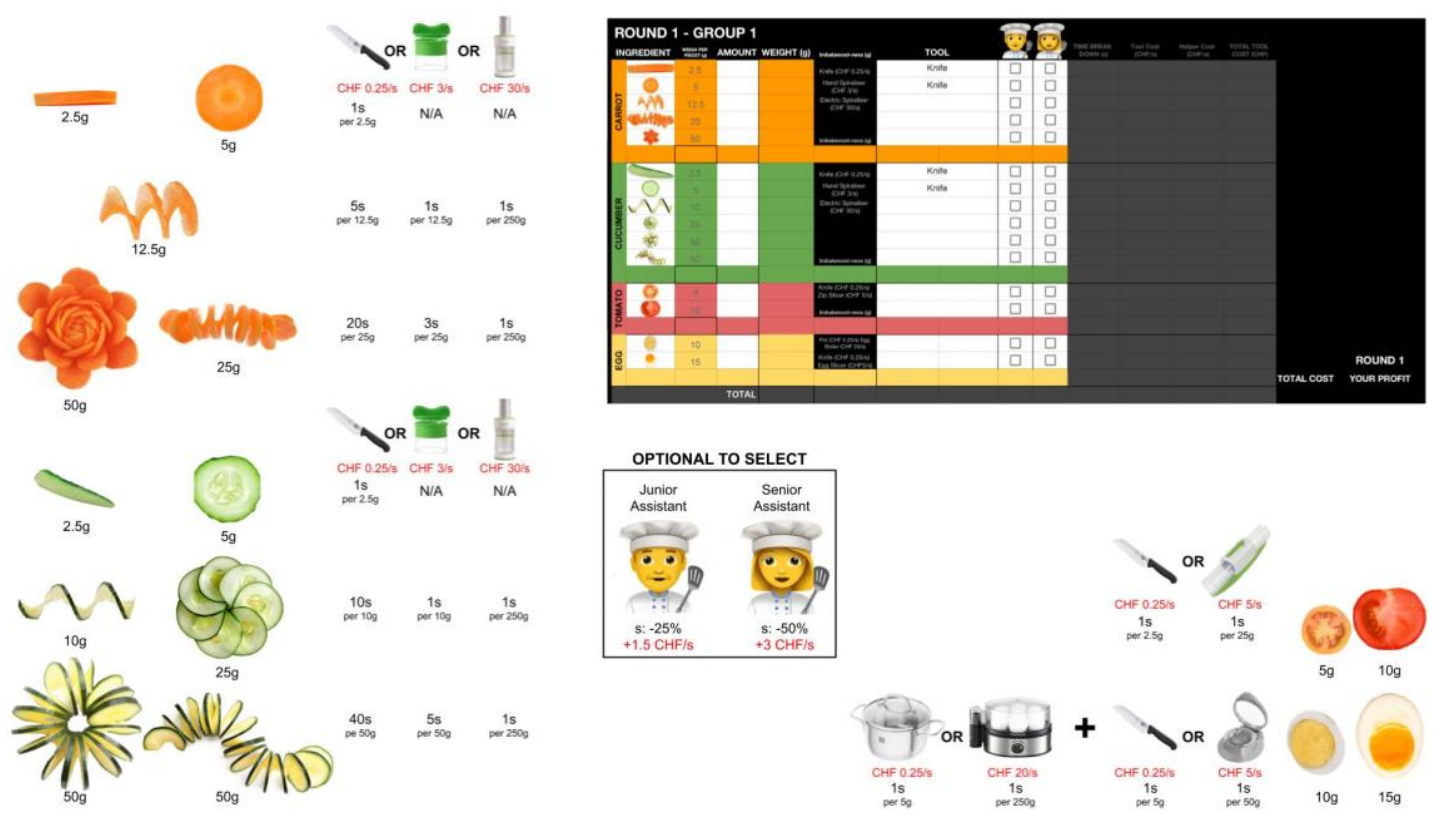

Figure 2: Available ingredients and the associated tools and calculation sheet in both rounds for each team to design the salad layout and price the process 

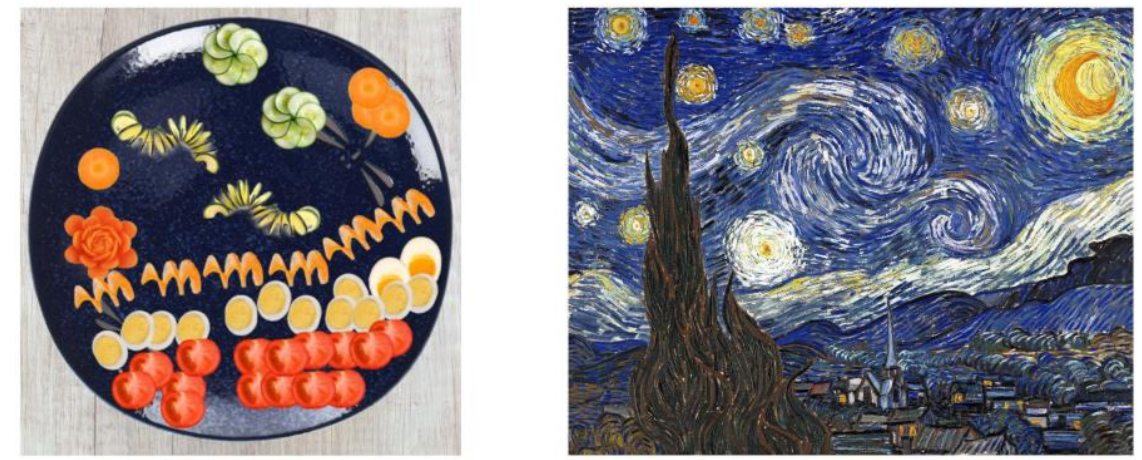

Figure 3: Design reference that mimics van Gogh's The Starry Night painting

Table 1 and Table 2 present the task details and the rundown of 15 mins duration in Round 1 and Round 2 respectively. The duration shaded in blue refers to the period when the players can actively work either on the Google Slides (as indicated with "SL") and/or on the Google Sheet (as indicated with "SH") to develop the salad design. While those shaded in green (as indicated with "View") refers to that the players can only passively observe the design processes conducted by their other teammates on both the Google Slides and the Google Sheet. The red thick vertical line indicates the design freeze cutoff time when the Artistic Chef and the Recipe Chef in each team can no longer continue their design development. In Round 1, the design process takes only 10 mins. The Executive Chef can only price the process after the design freeze; while in Round 2, the design process takes longer, with $15 \mathrm{mins}$, and all players have to stop their work at the design freeze. In Round 1, the Restaurant Owner is not allowed to provide any comment throughout the design process. While in Round 2, the Restaurant Owner can provide verbal feedback during the design process. At the end of the game, the Restaurant Owner in each team has to either approve or reject the salad design based on the customer's requirements and values to the project stakeholders. The design process in Round 1 is relatively sequential while that in Round 2 adopts integrated information and organisation in the design process.

Table 1: Task details and rundown of in total 15 mins duration in Round 1

\begin{tabular}{ccccc|c}
\hline Scope of work & Deliverables & Task owner & $\mathbf{5}$ min & $\mathbf{1 0 ~ m i n}$ & $\mathbf{1 5}$ min \\
\hline Layout concept design & The Starry Night & Artistic Chef & $\mathrm{SL}$ & View \\
Weights optimisation & Balanced weights & Recipe Chef & View & SL+SH & View \\
Process pricing & Good price & Executive Chef & View & SH \\
Design review & Customer's values & Restaurant Owner & View & View \\
\hline
\end{tabular}

Table 2: Task details and rundown of in total 15 mins duration in Round 2

\begin{tabular}{|c|c|c|c|c|c|}
\hline Scope of work & Deliverables & Task owner & $5 \min$ & $10 \min$ & $15 \min$ \\
\hline Layout concept design & The Starry Night & Artistic Chef & \multicolumn{3}{|c|}{ SL } \\
\hline Weights optimisation & Balanced weights & Recipe Chef & \multicolumn{3}{|c|}{$\mathrm{SL}+\mathrm{SH}$} \\
\hline Price optimisation & Good price & Executive Chef & \multicolumn{3}{|c|}{$\mathrm{SH}$} \\
\hline Design advice & Customer's values & Restaurant Owner & \multicolumn{3}{|c|}{ View + Comment } \\
\hline
\end{tabular}




\section{CASE STUDY RESULTS}

As a preliminary validation of the effictiveness of the TVDfDFAB game, this paper reports a case study taken from one playing of the game. The game was implemented on on $7^{\text {th }}$ December 2020 and played by 36 industry practitioners, researchers and master's degree students in the course "Lean, Integrated and Digital Project Delivery (LIDPD)" at ETH Zurich remotely in Switzerland. Their professional backgrounds include architecture, structural engineering and construction management. Table 3 presents the results of the case. ${ }^{4}$

The overall results firstly show that the mean $(\mu)$ and the standard deviation $(\sigma)$ of the total time in Round 2 is shorter than in Round 1. This was because the teams were more willing to adopt DFAB to foster automation. Secondly, the mean $(\mu)$ and the standard deviation $(\sigma)$ of the total cost in Round 2 is lower than that in Round 1. Thirdly, the mean $(\mu)$ profit in Round 2 is much higher than in Round 1, even though the profit in Round 1 was calculated in the way that the higher the cost, the higher the profit; while in Round 2, the profit was calculated by the set Target cost - CHF 200 - minus the total cost of the design delivered by each team. Last but not least, all design outputs in Round 2 have been approved by the Restaurant Owners base on the design performances such as the resemblance to The Starry Night painting. This shows that use of DFAB in TVD does not incur compromise in design of the aesthetic requirements, while achieving optimised values to stakeholders. The results of shorter time, lower cost and higher profit in Round 2 compared to Round 1 in this case results validate that this TVDfDFAB game helps to leverage the use of DFAB in TVD to maximise values in design.

Table 3: The results of ROUND 1 and ROUND 2 in the game's case study.

\begin{tabular}{c|cccc|cccc}
\hline & \multicolumn{3}{|c|}{ ROUND 1 - DBB approach } & \multicolumn{4}{c}{ ROUND 2 - TVD approach } \\
Group & $\begin{array}{c}\text { Total Cost } \\
\text { (CHF) }\end{array}$ & $\begin{array}{c}\text { Profit } \\
\text { (CHF) }\end{array}$ & $\begin{array}{c}\text { Total Time } \\
\text { (s) }\end{array}$ & $\begin{array}{c}\text { Design } \\
\text { approved? }\end{array}$ & $\begin{array}{c}\text { Total Cost } \\
\text { (CHF) }\end{array}$ & $\begin{array}{c}\text { Profit } \\
\text { (CHF) }\end{array}$ & $\begin{array}{c}\text { Total Time } \\
\text { (s) }\end{array}$ & $\begin{array}{c}\text { Design } \\
\text { approved? }\end{array}$ \\
\hline $\mathbf{1}$ & 217 & 11 & 11 & $\times$ & 176 & 24 & 44 & $\sqrt{ }$ \\
$\mathbf{2}$ & 321 & 16 & 37 & $\sqrt{ }$ & 185 & 15 & 36 & $\sqrt{ }$ \\
$\mathbf{3}$ & 160 & 8 & 48 & $\sqrt{ }$ & 176 & 24 & 40 & $\sqrt{ }$ \\
$\mathbf{4}$ & 327 & 16 & 78 & $\sqrt{ }$ & 192 & 8 & 26 & $\sqrt{ }$ \\
$\mathbf{5}$ & 183 & 9 & 39 & $\sqrt{ }$ & 159 & 41 & 31 & $\sqrt{ }$ \\
$\mathbf{6}$ & 167 & 8 & 29 & $\sqrt{ }$ & 160 & 40 & 33 & $\sqrt{ }$ \\
$\mathbf{7}$ & 254 & 13 & 60 & $\times$ & 160 & 40 & 67 & $\sqrt{ }$ \\
$\mathbf{8}$ & 194 & 10 & 61 & $\sqrt{ }$ & 152 & 48 & 28 & $\sqrt{ }$ \\
$\mathbf{9}$ & 272 & 14 & 172 & $\sqrt{ }$ & 190 & 10 & 64 & $\sqrt{ }$ \\
\hline $\boldsymbol{\mu}$ & 235 & 12 & 66 & & 172 & 28 & 41 & \\
$\mathbf{\sigma}$ & 67.8 & 3.4 & 45.8 & & 15.9 & 15.9 & 16.0 & \\
\hline
\end{tabular}

\footnotetext{
${ }^{4}$ The video recording of this case study dated $7^{\text {th }}$ December 2020 at ETH Zurich that demonstrates how this TVDfDFAB can be played via online platforms can be accessed here: https://www.youtube.com/watch?v=Nywx8C6QvjU
} 


\section{DISCUSSION}

\section{REFLECTION IN THE CONSTRUCTION INDUSTRY}

Although this game adopts the commercial kitchen scenario for the DFAB processes as explained above, the roles and the design processes in both rounds mimic those in the real-world construction projects (Table 4). The project values include aesthetic and functional requirements and the process costs as the design deliverables. Within a team, there is an overall goal, which is to deliver a design to customer's target values. However, each role has their different deliverables, scopes of work and timeline to contribute to the design as shown in Tables 1 and 2 above. Therefore, the players ought to communicate with their teammates to incorporate the tradeoffs such as costs, weights and asthetic requirements with the design during the design processes. In this game, the customer does not request for fast and automated process using DFAB, but the process time accounts for one fraction of the total cost. This mimics the scenario in a typical construction project, where DFAB technology and automation might not be requested by the project owners in the project brief, but the duration of the construction process would account for a fraction of the total cost in the project.

Furthermore, different from the Marshmallow game developed by Rybkowski et al. (2016), this TVDfDFAB game demonstrates how TVD should integrate downstream fabrication process information such as tools' capability, process cost and speed information upstreams into the design development. This is made possible only by integrated organisation during the design process. This game also demonstrates the challenges in the TVD process. While the Marshmallow game does not give specific scope of work to each player, in TVDfDFAB the four roles have different deliverables and scopes of work. In the DBB design process, the Artistic Chef in a team might have more freedom of design at the beginning, where the resemblance to The Starry Night was the only deliverable during the first 5mins. While in the TVD process, the Artistic Chef has to coordinate with the other teammates and the deliverable of the resemblance to The Starry Night might not be the first priority at the beginning of the round. This game shows that the TVD approach has its challenges in design coordination, which reflects real-world TVD processes. This TVDfDFAB game allows players to experience some "pros and cons" in DBB and TVD design processes in real-world construction projects.

Table 4: How the terms in this TVDfDFAB can be reflected in the construction industry

\begin{tabular}{cc|cc}
\hline TVDfDFAB Game & Construction & TVDfDFAB Game & Construction \\
\hline Artistic Chef & Design architect & Recipe Chef & Design engineer \\
Executive Chef & Contractor & Restaurant Owner & Design manager \\
\hline The Starry Night & Aesthetics challenge & Weight & Function/ performance \\
Kitchen tool & Fabrication machine & Design freeze & Tender \\
\hline
\end{tabular}

\section{LIMITATIONS}

This game is still in its early stage of development and requires further improvement. Amongst all, there are four key concerns, which the authors have conducted corresponding measures to address. Firstly, players with more DFAB experience in practice might perform better in the game. To address this concern, the game adopts a commercial kitchen scenario, where DFAB processes are relatively common in our daily life in many countries worldwide. Thus, DFAB practical experience is not a prerequisite; 
and this would not significantly impact on the performance of the game. The validation case also shows that the results of the game were independent upon the player's experience.

Secondly, players who are more familiar with digital software might perform better in the game. To address this concern, the authors chose two common-used cloud-based platforms - Google Slides and Google Sheet, where most players are familiar with their function and ways of usage in their daily routine. For example, the ingredient pieces were all pre-created. The Artistic Chef in each team merely has to select, drag and drop, or rotate, the pieces on the Google Slides. No particular DFAB or software skills are required. Also, the weight and cost can be calculated automatically on the Google Sheet. Thus, no mathematical or engineering calculations are required during the processes.

Thirdly, this game has simplified the design process compared to that in a typical construction project. The design process in each round does not yet cover all the requirements and constraints such as material selection, regulatory compliances etc. The authors in particular explore a game which magnifies the use of DFAB in TVD in the game, which has not yet been included in state-of-the-art TVD games. A future case study can further elaborate and include more criteria of the cost elements and design requirements and explore how players can undertake a more complex design process in both the DBB and TVD processes, which take not only DFAB process but also material requirements etc. into account.

Fourthly, this game is designed in the way that the players undertaken the DBB design process first and then the TVD process. It is possible that the players got familiar with the design criteria and the the workflow in Round 1 and therefore they might have learned from experience and performed better in Round 2. To address this concern, a control group experience can be conducted where the two rounds can be played by different teams in parallel simultaneously to investigate the potential impact of this limitation. This work requires further research to explore theoretically the use of DFAB in TVD and how this helps to maximise values to stakeholders in construction projects.

Finally, it should be noted that TVDfDFAB is intented to be a complementary game to the Marshmallow game that addresses some limitations - namely as an online format in consideration of DFAB. However, this is not to suggest that TVDfDFAB should be considered a superior or replacement for the Marshmallow game which has a strong trackrecord of success. Instead, TVDfDFAB is proposed as an alternative and educators should consider the benefits and tradeoffs of each game.

\section{CONCLUSION}

DFAB is emerging to foster automation and boost productivity in the construction industry (Agarwal et al. 2016). However, stakeholders find it hard to manage DFAB in construction projects because DFAB transforms the design process and requires downstream fabrication-related information and organisation to move upstream for design development (Carra et al. 2018). Discrete design and construction processes in traditional delivery models such as DBB hinders DFAB implementation ( $\mathrm{Ng}$ et al. 2020). TVD, which facilitates concurrent engineering and design-to-target-values, has been proposed as a potential design management approach to manage DFAB in the design process and maximise values to project stakeholders (Ng and Hall 2019). However, the use of DFAB in TVD in construction is still new in the industry. The authors of this work build on top of state-of-the-art TVD games such as the Marshmallow game developed by Rybkowski et al. (2016) to explore using TVD game to assist project stakeholders to 
comprehend TVD principles, so as to leverage the use of DFAB in TVD. This work presents a work-in-progress TVDfDFAB online game that allows players to consider DFAB processes in TVD. The game adopts a commercial kitchen scenario, where players conduct a salad design, which involves aesthetic, functional and cost challenges. Players form groups of four to conduct the design in Round 1 - DBB design process and Round 2 - TVD process successively. The roles, requirements, values and design processes mimic real-world construction projects. This work also presents a validation case played by 36 industry practitioners, researchers and students on $7^{\text {th }}$ December 2020 in Switzerland. The results show that TVD helps players to implement and manage DFAB to achieve shorter time, lower cost and higher profit without compromise in design of the aesthetic requirements while achieving optimised values to stakeholders. This work further illustrates four key concerns as limitations and future research is required to explore theoretically the use of DFAB in TVD in construction projects. All in all, this work contributes to the body of knowledge in learning and teaching TVD and technology adoption.

\section{ACKNOWLEDGMENTS}

The authors would like to thank Prof. Dr. Zofia Rybkowski, Mr. Alan Mossman and APLSO: Administering and Playing Lean Simulation Group for the feedback on the Alpha version played in early November 2020, and all the players who undertook the Bela version of the game on $7^{\text {th }}$ December 2020 at ETH Zurich in Switzerland for the case study validation.

\section{REFERENCES}

Agarwal, R., Chandrasekaran, S., \& Sridhar, M. (2016). Imagining construction's digital future.

Ballard, G., and Morris, P. H. (2010). "Maximizing owner value through Target Value Design.” 2010 AACE International Transactions: 1-16.

Ballard, G. (2011). "Target Value Design: Current Benchmark 1.0." Lean Construction Journal, 2011, 79-84.

Bock, T., \& Linner, T. (2015). Robot-Oriented Design. NY: Cambridge Univ. Press.

Bridgewater, C. (1993). "Principles of design for automation applied to construction tasks." Automation in Construction, 2(1), 57-64.

Carra, G., Argiolas, A., Bellissima, A., Niccolini, M., \& Ragaglia, M. (2018). "Robotics in the construction industry: State of the art and future opportunities." $35^{\text {th }}$ ISARC 2018 and International AEC/FM Hackathon: The Future of Building Things.

Hall, D. M., Algiers, A., \& Levitt, R. E. (2018). "Identifying the Role of Supply Chain Integration Practices in the Adoption of Systemic Innovations." Journal of Management in Engineering, 34(6), 04018030.

Hall, D. M., Whyte, J. K., \& Lessing, J. (2019). "Mirror-breaking strategies to enable digital manufacturing in Silicon Valley construction firms: a comparative case study." Construction Management and Economics, 0(0), 1-18.

Macomber, H., Howell, G., \& Barberio, J. (2007). "Target-Value Design: Nine Foundational Practices for Delivering Surprising Client Value." AIA Practice Management Digest, 2-4.

Miron, L. I. G., Kaushik, A., \& Koskela, L. (2015). „Target value design: The challenge of value generation." Proc. 23nd Ann. Conf. IGLC 23, 815-825. 
Ng, M. S., \& Hall, D. M. (2019). "Toward Lean Management for Digital Fabrication: a Review of the Shared Practices of Lean, DfMA and DFAB." Proc. 27th Ann. Conf. IGLC 2019, 725-736.

Ng, M. S., Bonanomi, M. M., Hall, D. M., \& Hackl, J. (2020). "Design for Digital Fabrication: an Industry needs Analysis of Collaboration Platforms and Integrated Management Processes." Proc. of the 37th ISARC, 318-325. Kitakyshu, Japan.

Rybkowski, Z. K. (2009). "The Application of Root Cause Analysis and Target Value Design to Evidence-Based Design in the Capital Planning of Healthcare Facilities." PhD Diss., Civil and Envir. Engrg, U.C. Berkeley.

Rybkowski, Z. K., Munankami, M. B., Shepley, M. M., \& Fernández-Solis, J. L. (2016). "Development and testing of a lean simulation to illustrate key principles of target value design: A first run study." 24th Ann. Conf. IGLC 2016., 133-142.

Tommelein, I. D. and Ballard, G. (2016). "Target Value Design: Introduction, Framework \& Current Benchmark." Lean Construction Institute, Arlington, VA.

World Economic Forum (2016). "Shaping the future of construction: a breakthrough in mindset and technology." World Economic Forum, Switzerland.

Zimina, D., Ballard, G., \& Pasquire, C. (2012). "Target value design: using collaboration and a lean approach to reduce construction cost." Construction Management and Economics, 30(5), 383-398. 